\title{
Polymer-supported Organotin Reagents for Regioselective Halogenation of Aromatic Amines
}

Jean-Mathieu Chrétien, ${ }^{a}$ Françoise Zammattio, ${ }^{* a}$ Erwan Le Grognec, ${ }^{a}$ Michaël Paris, ${ }^{b}$ Blanche Cahingt, ${ }^{c}$ Gilles Montavon, ${ }^{c}$ Jean-Paul Quintard ${ }^{*}, a$

${ }^{a}$ Laboratoire de Synthèse Organique, UMR CNRS 6513 \& FR CNRS 2465, Faculté des Sciences et des Techniques, 2 rue de la Houssinière, BP 92208, F-44322 Nantes cedex 3, France.

${ }^{b}$ Institut des Matériaux Jean Rouxel, UMR CNRS 6502, Faculté des Sciences et des Techniques, 2 rue de la Houssinière, BP 32229, F-44322 Nantes cedex 3, France.

${ }^{c}$ Subatech, UMR CNRS 6457, Ecole des Mines de Nantes, 4 rue Alfred Kastler, BP 20722, F-44307

Nantes cedex 3, France.

francoise.zammattio@univ-nantes.fr; jean-paul.quintard@univ-nantes.fr

\section{Supporting information}

\section{Table of contents}

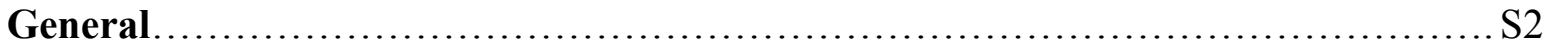

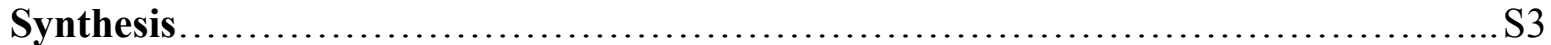

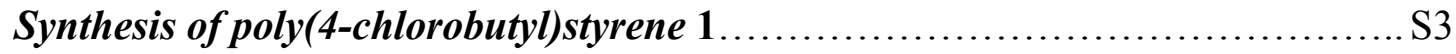

Synthesis of poly[4-(dibutylphenylstannyl)butyl]styrene 2.........................S3

Synthesis of poly[4-(dibutyliodostannyl)butyl]styrene 3a............................. 4

General procedure for the bromination of aromatic amines...................... S4

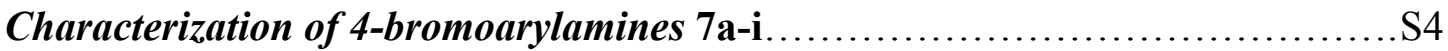

General procedure for the iodination of aromatic amines.......................... 8

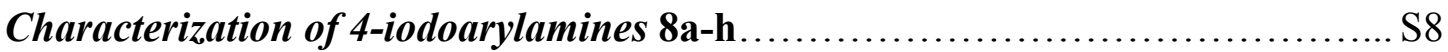




\section{General}

All starting materials were obtained from commercial suppliers and used without further purification. Dibutylphenyltin hydride was obtained using an already described procedure. ${ }^{\mathrm{s} 1}$ Diethyl ether and THF were distilled from sodium/benzophenone ketyl and cyclohexane was distilled over calcium hydride.

${ }^{1} \mathrm{H}$ and ${ }^{13} \mathrm{C}$ NMR spectra were recorded on a spectrometer operating at $300 \mathrm{MHz}$ for ${ }^{1} \mathrm{H}$ and 75.5 $\mathrm{MHz}$ for ${ }^{13} \mathrm{C}$ in $\mathrm{CDCl}_{3}$ solution. Chemical shifts $(\delta)$ are expressed in ppm downfield to the tetramethylsilane (TMS) as the internal standard and coupling constants $(J)$ are expressed in Hertz.

Solid state MAS NMR experiments were performed at room temperature on a spectrometer operating at $186.5 \mathrm{MHz}$ for ${ }^{119} \mathrm{Sn}$ using a $4 \mathrm{~mm}$ double-bearing probehead. ${ }^{119} \mathrm{Sn}$ MAS spectra were acquired with ${ }^{1} \mathrm{H}$ TPPM decoupling ${ }^{\mathrm{s} 2}$ during acquisition and a MAS frequency of $10 \mathrm{kHz}$. Repetition time was set to $20 \mathrm{~s}$ for quantitative purpose since ${ }^{119 \mathrm{Sn}} \mathrm{T}_{1}$ were measured in the order of $3 \mathrm{~s}$. Spectra were referenced to $\mathrm{Me}_{4} \mathrm{Sn}$ using $\mathrm{Ph}_{4} \mathrm{Sn}$ as a secondary reference $(-97.35 \mathrm{ppm}){ }^{\mathrm{s} 3}$ Mass spectra were recorded in EI $(70 \mathrm{eV})$ in GC-MS mode: the $\mathrm{m} / \mathrm{z}$ values are given for meaningful signals and in the case of isotopic patterns containing $\mathrm{Br}$, the given abundance refers to the higher peak.

IR spectra were recorded on a FT-IR spectrometer from $400 \mathrm{mg}$ dry KBr pellets with $4 \mathrm{mg}$ of substance or $10 \mathrm{mg}$ of polymer. Polystyrene bands $\left(\mathrm{cm}^{-1}\right):{ }^{\mathrm{s} 4}$ aromatic C-H stretchings, 3082, 3059, 3025; aliphatic C-H stretchings, 2921, 2850; weak overtone bands, 1942, 1870, 1802, 1719 (monosubstituted aromatic rings); aromatic C-C stretchings: 1601, 1586, 1493, 1452; weak inplane aromatic $\mathrm{C}-\mathrm{H}$ bending band, 1028; out-of-plane aromatic $\mathrm{C}-\mathrm{H}$ bending bands, 759, 698 (monosubstituted aromatic rings).

Elemental analyses were carried out by the CNRS Analysis Central Laboratory, Vernaison, France, in order to calculate tin and halogen loadings on the resins.

ICP-MS analyses were performed with an ICP operating at $1350 \mathrm{~W}$ and all parameters were optimized to obtain the maximum sensitivity (ions optics, flow rates, glassware position...).

(s1) Ruel, G.; The, N. K.; Dumartin, G.; Delmond, B.; Pereyre, M. J. Organomet. Chem. 1993, 444, C18-C20.

(s2) Bennett, A. E.; Rienstra, C. M.; Auger, M.; Lakshmi, K. V.; Griffin. R. G. J. Chem. Phys. 1995, 103, 69516958.

(s3) Dumartin, G.; Kharboutli, J.; Delmond, B.; Pereyre, M.; Biesemans, M.; Gielen, M.; Willem, R. Organometallics 1996, 15, 19-23.

(s4) Mercier, F. A. G.; Biesemans, M.; Altmann, R.; Willem, R.; Pintelon, R.; Schoukens, J.; Delmond, B.; Dumartin, G. Organometallics 2001, 20, 958-962. 
Nebulization was performed with a nebulizer working at around $1 \mathrm{~mL} \cdot \mathrm{min}^{-1}$. A fully quantified analytical method was set up, using 8 points of calibration between 0 and $1 \mathrm{ppb}$ of tin. The material has been dissolved in $3 \mathrm{~mL}$ of a mixture of $2 \%$ nitric acid and acetone and interferences of the matrix have been studied in order to correct the analytical results. No internal reference was used but the stability over time had been checked up every 5 samples, and memory effect had been checked up between each sample.

\section{Synthesis}

\section{Synthesis of poly(4-chlorobutyl)styrene $1^{\text {s1 }}$}

To a suspension of Amberlite XE 305 (6.0 g) in dry cyclohexane (15 mL) were added successively, under argon, TMEDA $(8.7 \mathrm{~mL}, 57.7 \mathrm{mmol})$ and a solution of $n$-butyllithium (2.5 M in hexanes, $27.7 \mathrm{~mL}, 69.2 \mathrm{mmol}$ ). The reaction mixture was heated at $65^{\circ} \mathrm{C}$ for $4 \mathrm{~h}$. The resin was then washed under argon with dry cyclohexane $(8 \times 20 \mathrm{~mL})$ and this procedure (TMEDA, $n$ butyllithium) was repeated once. The orange resulting lithiated polymer was then washed under argon with dry THF $(12 \times 20 \mathrm{~mL})$. 1-Bromo-4-chlorobutane $(7.9 \mathrm{~mL}, 68.6 \mathrm{mmol})$ was added, at $0^{\circ} \mathrm{C}$ under argon, on the lithiated polymer suspension in dry THF $(50 \mathrm{~mL})$. The mixture was stirred at room temperature for $18 \mathrm{~h}$ then the resulting polymer was successively washed with a mixture of 1:1 THF/ $\mathrm{H}_{2} \mathrm{O}(40 \mathrm{~mL})$, THF $(6 \times 40 \mathrm{~mL})$, absolute ethanol $(4 \times 40 \mathrm{~mL})$ and dried in vacuo $(0.5 \mathrm{mbar})$ at $60^{\circ} \mathrm{C}$ for $5 \mathrm{~h}$. Polymer 1 was obtained as a white resin $(7.8 \mathrm{~g})$ and was found to contain $2.21 \mathrm{mmol}$ of $\mathrm{C}-\mathrm{Cl} / \mathrm{g}$. Anal. Found: C, 82.59; H, 7.72; Cl, 7.86; Br, 0.43. IR (KBr) $v$ 3082, 3059, 3025, 2921, 2850, 1942, 1870, 1802, 1719, 1601, 1586, 1493, 1452, 1028, 759, 698, $650,539 \mathrm{~cm}^{-1}$.

\section{Synthesis of poly[4-(dibutylphenylstannyl)butyl]styrene $2^{\text {s1 }}$}

To a solution of lithium diisopropylamide $(30.5 \mathrm{mmol})$ in dry THF was slowly added, at $0^{\circ} \mathrm{C}$ under argon, dibutylphenyltin hydride $(8.62 \mathrm{~g}, 27.7 \mathrm{mmol})$. The resulting mixture was stirred for $1 \mathrm{~h}$ at $0^{\circ} \mathrm{C}$ and subsequently added at $0^{\circ} \mathrm{C}$ under argon to the dry polymer $3(6.0 \mathrm{~g})$. The mixture was allowed to warm up to room temperature and stirred for $18 \mathrm{~h}$. The resulting polymer was successively washed with a mixture of $1: 1 \mathrm{THF} / \mathrm{H}_{2} \mathrm{O}(60 \mathrm{~mL})$, THF $(6 \times 60 \mathrm{~mL})$, absolute ethanol $(4 \times 60 \mathrm{~mL})$ and dried in vacuo $(0.5 \mathrm{mbar})$ at $60^{\circ} \mathrm{C}$ for $5 \mathrm{~h}$. Polymer 2 was obtained as a white resin (10.4 g) and was found to contain $1.33 \mathrm{mmol}$ of $\mathrm{Sn} / \mathrm{g}$. Anal. Found: C, 74.87; H, 7.12; Sn, 15.76; Cl, <0.20. ${ }^{119}$ Sn MAS NMR (186.5 MHz) $\delta_{\mathrm{Sn}}-20$ ppm; IR (KBr) v 3082, 3059, 3025, 2921, 2850, 1942, 1870, 1802, 1719, 1601, 1586, 1493, 1452, 1073, 1028, 759, 726, $698 \mathrm{~cm}^{-1}$. 


\section{Synthesis of poly[4-(dibutyliodostannyl)butyl]styrene $3 \mathrm{a}^{\mathrm{s} 1}$}

A solution of iodine $(3.81 \mathrm{~g}, 15.0 \mathrm{mmol})$ in absolute ethanol $(50 \mathrm{~mL})$ was added to the polymer 2 $(10.0 \mathrm{~g})$ and the resulting mixture was stirred at $60^{\circ} \mathrm{C}$ for $18 \mathrm{~h}$ in the dark. The polymer was successively washed with a mixture of $1: 1 \mathrm{THF} /$ aqueous $\mathrm{Na}_{2} \mathrm{~S}_{2} \mathrm{O}_{3}(60 \mathrm{~mL})$, THF $(6 \times 60 \mathrm{~mL})$, absolute ethanol $(4 \times 60 \mathrm{~mL})$ and dried in vacuo $(0.5 \mathrm{mbar})$ at $60^{\circ} \mathrm{C}$ for $5 \mathrm{~h}$. Polymer $3 \mathbf{a}$ was obtained as a pale yellow resin $(10.7 \mathrm{~g})$ and was found to contain $1.16 \mathrm{mmol}$ of $\mathrm{Sn}-\mathrm{I} / \mathrm{g}$. Anal. Found: C, 63.22; H, 7.00; Sn, 14.30; I, 14.78. ${ }^{119}$ Sn MAS NMR (186.5 MHz) $\delta_{\text {Sn }} 104$ ppm; IR $(\mathrm{KBr})$ v 3082, 3059, 3025, 2921, 2850, 1942, 1870, 1802, 1719, 1601, 1586, 1493, 1452, 1073 , $1028,759,698 \mathrm{~cm}^{-1}$.

\section{General procedure for the bromination of aromatic amines.}

To a solution of aromatic amine $4(2.4 \mathrm{mmol})$ in anhydrous diethyl ether $(32 \mathrm{~mL})$ was added dropwise, at $-78^{\circ} \mathrm{C}$ under argon, a solution of $n$-butyllithium $(2.5 \mathrm{M}$ in hexanes, $0.96 \mathrm{~mL}, 2.4$ mmol). The resulting mixture was stirred for $1 \mathrm{~h}$ at $-78^{\circ} \mathrm{C}$ and then added to the dry polymer $3 \mathbf{a}$ $(2.07 \mathrm{~g}, 2.4 \mathrm{mmol})$. The resulting heterogeneous mixture was stirred for $18 \mathrm{~h}$ at room temperature before adding bromine $\left(1.0 \mathrm{M}\right.$ in $\left.\mathrm{CH}_{2} \mathrm{Cl}_{2}, 2.4 \mathrm{~mL}, 2.4 \mathrm{mmol}\right)$ at $-78^{\circ} \mathrm{C}$. The mixture was stirred for $1 \mathrm{~h}$ at $-78^{\circ} \mathrm{C}$ and $1 \mathrm{~h}$ at room temperature, and the polymer was filtered. The resin was at first washed with diethyl ether $(6 \times 40 \mathrm{~mL})$, then with THF $(6 \times 40 \mathrm{~mL})$ and absolute ethanol $(4 \times 40$ $\mathrm{mL})$, and dried in vacuo $(0.5 \mathrm{mbar})$ at $60^{\circ} \mathrm{C}$ for $5 \mathrm{~h}$ before reuse. Polymer $\mathbf{3 b}$ was obtained as a blue-brown resin and was found to contain $1.31 \mathrm{mmol}$ of $\mathrm{Sn}-\mathrm{Br} / \mathrm{g}$. Anal. Found: C, 65.64; H, 7.38; Sn, 15.54; Br, 10.80; I, 0.46. ${ }^{119} \mathrm{Sn}$ MAS NMR (186.5 MHz) $\delta_{\text {Sn }} 157$ ppm; IR (KBr) v3082, 3059, 3025, 2921, 2851, 1943, 1870, 1800, 1719, 1601, 1586, 1493, 1452, 1073, 1028, 759, 698

$\mathrm{cm}^{-1}$. The ethereal filtrate was then successively washed with a saturated solution of $\mathrm{NaHCO}_{3}(50$ $\mathrm{mL})$ and brine $(50 \mathrm{~mL})$, dried over $\mathrm{MgSO}_{4}$ and concentrated in vacuo. The crude product was purified by chromatography on silica gel.

\section{Characterization of 4-bromoarylamines $7 \mathrm{a}-\mathbf{i}$}

All compounds $\mathbf{7 a - i}$ were obtained following the general procedure for the bromination of aromatic amines.

4-bromoaniline $7 \mathbf{a} .{ }^{55}$ Compound $7 \mathbf{a}$ was obtained from aniline $\mathbf{4 a}(2.43 \mathrm{mmol})$ as a pale brown solid after chromatography (9:1, petroleum ether/AcOEt, $\left.1 \% \mathrm{Et}_{3} \mathrm{~N}, \mathrm{Rf}=0.18\right): 317 \mathrm{mg}, 76 \%$; $\mathrm{mp}$

(s5) Berrier, C.; Jacquesy, J. C.; Renoux, A. Bull. Soc. Chim. Fr. 1990, 127, 93-97. 
62-63 ${ }^{\circ} \mathrm{C} ;{ }^{1} \mathrm{H}$ NMR $\left(300 \mathrm{MHz}, \mathrm{CDCl}_{3}\right) \delta_{\mathrm{H}} 3.66$ (br s, $\left.2 \mathrm{H}\right), 6.56(\mathrm{dd}, J=6.6 \mathrm{~Hz}, J=2.1 \mathrm{~Hz}, 2 \mathrm{H})$, $7.23(\mathrm{dd}, J=6.6 \mathrm{~Hz}, J=2.1 \mathrm{~Hz}, 2 \mathrm{H}) ;{ }^{13} \mathrm{C} \mathrm{NMR}\left(75.5 \mathrm{MHz}, \mathrm{CDCl}_{3}\right) \delta_{\mathrm{C}} 110.2,116.7,132.0$, 145.4; MS-EI m/z (relative intensity) 173/171 (100), 92 (86), 91 (10), 86.5/85.5 (10), 65 (76), 63 (15), 45.5 (14), 39 (18); IR (KBr) v3474, 3283, 1613, 1592, 1488, 1287, 827, 605, $503 \mathrm{~cm}^{-1}$.

2,4-dibromoaniline. ${ }^{\mathrm{s} 6}{ }^{1} \mathrm{H} \mathrm{NMR}\left(300 \mathrm{MHz}, \mathrm{CDCl}_{3}\right) \delta_{\mathrm{H}} 4.10$ (br s, 2H), $6.64(\mathrm{~d}, J=8.4 \mathrm{~Hz}, 1 \mathrm{H})$, $7.20(\mathrm{dd}, J=8.4 \mathrm{~Hz}, J=2.4 \mathrm{~Hz}, 1 \mathrm{H}), 7.53(\mathrm{~d}, J=2.4 \mathrm{~Hz}, 1 \mathrm{H})$; MS-EI $m / z$ (relative intensity) 253/251/249 (100), 172/170 (20), 91 (24), 90 (37), 63 (26).

2,4,6-tribromoaniline. ${ }^{\mathrm{s}}{ }^{1} \mathrm{H} \mathrm{NMR}\left(300 \mathrm{MHz}, \mathrm{CDCl}_{3}\right) \delta_{\mathrm{H}} 4.57$ (br s, 2H), $7.51(\mathrm{~s}, 2 \mathrm{H})$; MS-EI $\mathrm{m} / z$ (relative intensity) 333/331/329/327 (100), 252/250/248 (34), 171/169 (23), 170/168 (37), 166.5/165.5/164.5/163.5 (15), 90 (41), 89 (27), 85.5/84.5 (25), 63 (49), 62 (54), 61 (32), 52 (22), $44.5(30)$.

4-bromo-2-nitroaniline $7 \mathbf{b} .{ }^{\mathrm{s} 7}$ Compound $\mathbf{7 b}$ was obtained from 2-nitroaniline $\mathbf{4 b}(2.34 \mathrm{mmol})$ as an orange solid after chromatography (8:2, petroleum ether/AcOEt, $\left.1 \% \mathrm{Et}_{3} \mathrm{~N}, \mathrm{Rf}=0.33\right): 281$ mg, 55\%; mp 113-114 ${ }^{\circ} \mathrm{C} ;{ }^{1} \mathrm{H}$ NMR $\left(300 \mathrm{MHz}, \mathrm{CDCl}_{3}\right) \delta_{\mathrm{H}} 6.09$ (br s, 2H), 6.72 (d, $J=8.7 \mathrm{~Hz}$, $1 \mathrm{H}), 7.43(\mathrm{dd}, J=8.7 \mathrm{~Hz}, J=2.4 \mathrm{~Hz}, 1 \mathrm{H}), 8.27(\mathrm{~d}, J=2.4 \mathrm{~Hz}, 1 \mathrm{H}) ;{ }^{13} \mathrm{C} \mathrm{NMR}(75.5 \mathrm{MHz}$, $\left.\mathrm{CDCl}_{3}\right) \delta_{\mathrm{C}} 107.9,120.3,128.4,132.4,138.5,143.5$; MS-EI $\mathrm{m} / z$ (relative intensity) 218/216 (100), 188/186 (8), 172/170 (45), 145/143 (19), 91 (56), 90 (52), 64 (25), 63 (46), 52 (24); IR (KBr) v $3472,3342,3093,1638,1559,1500,1456,1403,1365,1338,1249,886,814 \mathrm{~cm}^{-1}$.

4-bromo-2-iodoaniline 7c. Compound 7c was obtained from 2-iodoaniline 4c (2.48 $\mathrm{mmol})$ as a brown solid after chromatography (8:2, petroleum ether/AcOEt, $\left.1 \% \mathrm{Et}_{3} \mathrm{~N}, \mathrm{Rf}=0.55\right): 340 \mathrm{mg}$, 46\%; mp 70-71 ${ }^{\circ} \mathrm{C} ;{ }^{1} \mathrm{H}$ NMR $\left(300 \mathrm{MHz}, \mathrm{CDCl}_{3}\right) \delta_{\mathrm{H}} 4.11$ (br s, $\left.2 \mathrm{H}\right), 6.62(\mathrm{~d}, J=8.4 \mathrm{~Hz}, 1 \mathrm{H}), 7.22$ $(\mathrm{dd}, J=8.4 \mathrm{~Hz}, J=2.1 \mathrm{~Hz}, 1 \mathrm{H}), 7.73(\mathrm{~d}, J=2.1 \mathrm{~Hz}, 1 \mathrm{H}) ;{ }^{13} \mathrm{C} \mathrm{NMR}\left(75.5 \mathrm{MHz}, \mathrm{CDCl}_{3}\right) \delta_{\mathrm{C}} 84.1$, 109.9, 115.5, 132.1, 140.4, 145.9; MS-EI m/z (relative intensity) 299/297 (100), 172/170 (36), 145/143 (14), 127 (10), 91 (65), 90 (54), 64 (42), 63 (86), 62 (33), 52 (42), 41 (19), 38 (23); IR $(\mathrm{KBr}) v 3384,3288,3175,3070,3046,1617,1573,1475,1386,1022,871,810 \mathrm{~cm}^{-1}$.

2,4-dibromo-6-iodoaniline. ${ }^{1} \mathrm{H}$ NMR $\left(300 \mathrm{MHz}, \mathrm{CDCl}_{3}\right) \delta_{\mathrm{H}} 7.54\left(\mathrm{~d},{ }^{4} J=2.1 \mathrm{~Hz}, 1 \mathrm{H}\right), 7.70\left(\mathrm{~d},{ }^{4} J\right.$ $=2.1 \mathrm{~Hz}, 1 \mathrm{H})$.

(s6) Choi, H. Y.; Chi, D. Y. J. Am. Chem. Soc. 2001, 123, 9202-9203.

(s7) Dirk, S. M.; Tour, J. M. Tetrahedron 2003, 59, 287-293. 
4-bromo-2-fluoroaniline 7d. ${ }^{88}$ Compound 7d was obtained from 2-fluoroaniline 4d (2.4 mmol) as an orange solid after chromatography (9:1, petroleum ether/AcOEt, $\left.1 \% \mathrm{Et}_{3} \mathrm{~N}, \mathrm{Rf}=0.3\right): 320$ mg, 70\%; ${ }^{1} \mathrm{H}$ NMR (300 MHz, $\left.\mathrm{CDCl}_{3}\right) \delta_{\mathrm{H}} 3.54$ (br s, 2H), $6.58(\mathrm{dd}, J=8.5 \mathrm{~Hz}, J=9.3 \mathrm{~Hz}, 1 \mathrm{H})$, $6.98(\mathrm{ddd}, J=2.1 \mathrm{~Hz}, J=8.5 \mathrm{~Hz}, J=1.1 \mathrm{~Hz}, 1 \mathrm{H}), 7.06(\mathrm{dd}, J=2.1 \mathrm{~Hz}, J=10.5 \mathrm{~Hz}, 1 \mathrm{H})$.

4-bromo-2-methoxyaniline 7e. Compound 7e was obtained from 2-methoxyaniline 4e (1.2 mmol) as a brown solid after chromatography $\left(85: 15\right.$, petroleum ether/AcOEt, $1 \% \mathrm{Et}_{3} \mathrm{~N}, \mathrm{Rf}=$ 0.31): $178 \mathrm{mg}, 73 \%$; mp 60-61 ${ }^{\circ} \mathrm{C} ;{ }^{1} \mathrm{H}$ NMR (300 MHz, $\left.\mathrm{CDCl}_{3}\right) \delta_{\mathrm{H}} 3.78$ (br s, 2H), 3.84 (s, 3H), $6.58(\mathrm{~d}, J=8.4 \mathrm{~Hz}, 1 \mathrm{H}), 6.88-6.91(\mathrm{~m}, 2 \mathrm{H}) ;{ }^{13} \mathrm{C} \mathrm{NMR}\left(75.5 \mathrm{MHz}, \mathrm{CDCl}_{3}\right) \delta_{\mathrm{C}} 55.7,109.6,113.7$, 115.7, 123.7, 135.3, 147.9; MS-EI $m / z$ (relative intensity) 203/201 (92), 188/186 (100), 160/158 (41), 107 (17), 93 (7), 91 (7), 90 (8), 79 (24), 78 (26), 63 (10), 52 (35), 51 (25); IR (KBr) v 3248, 3298, 3180, 3103, 2958, 2927, 2904, 2854, 2830, 1615, 1583, 1504, 1283, 1227, 1029, 860, 830, $809 \mathrm{~cm}^{-1}$.

2,4-dibromo-6-methoxyaniline. ${ }^{1} \mathrm{H}$ NMR $\left(300 \mathrm{MHz}, \mathrm{CDCl}_{3}\right) \delta_{\mathrm{H}} 3.84$ (s, 3H), 4.20 (br s, 2H), $6.82(\mathrm{~d}, J=2.1 \mathrm{~Hz}, 1 \mathrm{H}), 7.18(\mathrm{~d}, J=2.1 \mathrm{~Hz}, 1 \mathrm{H})$; MS-EI $m / z$ (relative intensity) 283/281/279 (85), 268/266/264 (100), 240/238/236 (24), 159/157 (6), 158/156 (5), 93 (7), 90 (10), 78 (33), 75 (15), $51(20)$.

4-bromo-3-methoxyaniline 7f. Compound 7f was obtained from 3-methoxyaniline 4f (1.2 mmol) as a brown solid after chromatography $\left(75: 25\right.$, petroleum ether/AcOEt, $1 \% \mathrm{Et}_{3} \mathrm{~N}, \mathrm{Rf}=$ 0.17): $110 \mathrm{mg}, 45 \%$; mp 93-94 ${ }^{\circ} \mathrm{C} ;{ }^{1} \mathrm{H}$ NMR (300 MHz, $\left.\mathrm{CDCl}_{3}\right) \delta_{\mathrm{H}} 3.71$ (br s, 2H), 3.83 (s, 3H), $6.18(\mathrm{dd}, J=8.4 \mathrm{~Hz}, J=2.7 \mathrm{~Hz}, 1 \mathrm{H}), 6.25(\mathrm{~d}, J=2.7 \mathrm{~Hz}, 1 \mathrm{H}), 7.24(\mathrm{~d}, J=8.4 \mathrm{~Hz}, 1 \mathrm{H}) ;{ }^{13} \mathrm{C}$ NMR $\left(75.5 \mathrm{MHz}, \mathrm{CDCl}_{3}\right) \delta_{\mathrm{C}} 56.0,99.5,99.6,108.5,133.4,147.2$, 156.4; MS-EI $\mathrm{m} / z$ (relative intensity) 203/201 (100), 160/158 (23), 122 (22), 107 (13), 94 (25), 93 (20), 92 (91), 79 (23), 78 (22), 65 (24), 52 (20), 51 (17).

2-bromo-5-methoxyaniline. ${ }^{1} \mathrm{H}$ NMR $\left(300 \mathrm{MHz}, \mathrm{CDCl}_{3}\right) \delta_{\mathrm{H}} 3.75(\mathrm{~s}, 3 \mathrm{H}), 4.07$ (br s, 2H), 6.23 $(\mathrm{dd}, J=8.7 \mathrm{~Hz}, J=2.7 \mathrm{~Hz}, 1 \mathrm{H}), 6.32(\mathrm{~d}, J=2.7 \mathrm{~Hz}, 1 \mathrm{H}), 7.27(\mathrm{~d}, J=8.4 \mathrm{~Hz}, 1 \mathrm{H})$; MS-EI $m / z$ (relative intensity) 203/201 (100), 173/171 (15), 160/158 (22), 94 (23), 93 (72), 92 (22), 79 (17), 78 (16), 61 (17), 52 (23), 51(18).

2,4-dibromo-5-methoxyaniline. MS-EI $m / z$ (relative intensity) 283/281/279 (100), 240/238/236 (24), 172/170 (16), 93 (19), 90 (13), 78 (23), 52 (12), 51 (13).

(s8) Data in good agreement with those from Sigma-Aldrich. 
2-bromo-4-methoxyaniline 7g. ${ }^{\mathrm{s} 9}$ Compound $\mathbf{7 g}$ was obtained from 4-methoxyaniline $\mathbf{4 g}$ (1.2 mmol) as a brown liquid after chromatography $\left(85: 15\right.$, petroleum ether/AcOEt, $1 \% \mathrm{Et}_{3} \mathrm{~N}, \mathrm{Rf}=$ 0.28): $129 \mathrm{mg}, 53 \% ;{ }^{1} \mathrm{H}$ NMR (300 MHz, $\left.\mathrm{CDCl}_{3}\right) \delta_{\mathrm{H}} 3.72(\mathrm{~s}, 3 \mathrm{H}), 3.80(\mathrm{br} \mathrm{s}, 2 \mathrm{H}), 6.72(\mathrm{~m}, 2 \mathrm{H})$, $7.00(\mathrm{~d}, J=2.4 \mathrm{~Hz}, 1 \mathrm{H}) ;{ }^{13} \mathrm{C} \mathrm{NMR}\left(75.5 \mathrm{MHz}, \mathrm{CDCl}_{3}\right) \delta_{\mathrm{C}} 55.9,109.6,115.1,116.7,117.5$, 138.0, 152.7; MS-EI m/z (relative intensity) 203/201 (66), 188/186 (100), 160/158 (22), 90 (7), 79 (19), 78 (15), 52 (44); IR (neat) v 3442, 3358, 3203, 2999, 2949, 2832, 1623, 1600, 1499, $1440,1275,1230,1212,1037,865 \mathrm{~cm}^{-1}$.

2,6-dibromo-4-methoxyaniline. ${ }^{1} \mathrm{H}$ NMR $\left(300 \mathrm{MHz}, \mathrm{CDCl}_{3}\right) \delta_{\mathrm{H}} 3.72(\mathrm{~s}, 3 \mathrm{H}), 4.19$ (br s, 2H), 7.01 (s, 2H); MS-EI m/z (relative intensity) 283/281/279 (52), 268/266/264 (100), 240/238/236 (10), $78(26), 52(20)$.

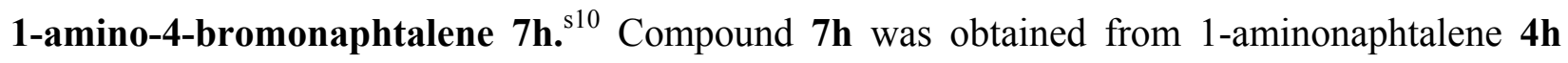
$(2.34 \mathrm{mmol})$ as a purple solid after chromatography $\left(8: 2\right.$, petroleum ether/AcOEt, $1 \% \mathrm{Et}_{3} \mathrm{~N}, \mathrm{Rf}=$ 0.16): $218 \mathrm{mg}, 42 \%$; mp 101-102 ${ }^{\circ} \mathrm{C} ;{ }^{1} \mathrm{H}$ NMR $\left(300 \mathrm{MHz}, \mathrm{CDCl}_{3}\right) \delta_{\mathrm{H}} 4.18$ (br s, 2H), 6.66 (d, $J=$ $8.1 \mathrm{~Hz}, 1 \mathrm{H}), 7.48-7.61(\mathrm{~m}, 3 \mathrm{H}), 7.81(\mathrm{~d}, J=8.4 \mathrm{~Hz}, 1 \mathrm{H}), 8.20(\mathrm{~d}, J=8.1 \mathrm{~Hz}, 1 \mathrm{H}) ;{ }^{13} \mathrm{C} \mathrm{NMR}$ $\left(75.5 \mathrm{MHz}, \mathrm{CDCl}_{3}\right) \delta_{\mathrm{C}} 110.2,111.4,121.2,124.8,125.6,127.2,127.8,130.1,132.3,142.1$; MSEI $m / z$ (relative intensity) 223/221 (100), 142 (21), 141 (5), 140 (11), 115 (67), 114 (15), 111.5/110.5 (8), 70.5 (19); IR (KBr) v3417, 3316, 1626, 1514, 1450, 1372, 1273, $807 \mathrm{~cm}^{-1}$.

1-amino-2,4-dibromonaphtalene. ${ }^{1} \mathrm{H}$ NMR $\left(300 \mathrm{MHz}, \mathrm{CDCl}_{3}\right) \delta_{\mathrm{H}} 4.67$ (br s, 2H), 7.51-7.62 (m, 2H), 7.78-7.81 (m, 2H), $8.17(\mathrm{dd}, J=8.7 \mathrm{~Hz}, J=1.2 \mathrm{~Hz}, 1 \mathrm{H})$.

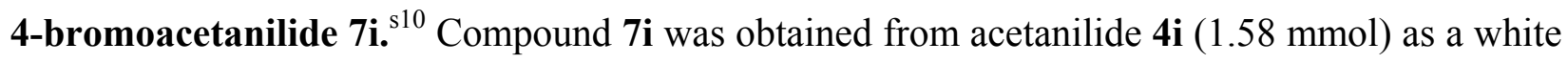
solid after chromatography (6:4, petroleum ether/AcOEt, 1\% $\left.\mathrm{Et}_{3} \mathrm{~N}, \mathrm{Rf}=0.18\right): 229 \mathrm{mg}, 68 \%$; $\mathrm{mp}$ $167-168^{\circ} \mathrm{C} ;{ }^{1} \mathrm{H}$ NMR $\left(300 \mathrm{MHz}, \mathrm{CDCl}_{3}\right) \delta_{\mathrm{H}} 2.17(\mathrm{~s}, 3 \mathrm{H}), 7.20(\mathrm{br} \mathrm{s}, 1 \mathrm{H}), 7.41(\mathrm{~s}, 4 \mathrm{H}) ;{ }^{13} \mathrm{C} \mathrm{NMR}$ $\left(75.5 \mathrm{MHz}, \mathrm{CDCl}_{3}\right) \delta_{\mathrm{C}} 24.6,116.9,121.4,132.0,136.9,168.3$; MS-EI $m / z$ (relative intensity) 215/213 (23), 173/171 (87), 92 (56), 91 (20), 65 (44), 64 (25), 63 (34), 43 (100), 39 (20); IR $(\mathrm{KBr}) v 3304,3265,3193,3123,1671,1604,1588,1535,1488,1393,1370,1314,1260$, 823, $745 \mathrm{~cm}^{-1}$.

(s9) Tidwell, J. H.; Buchwald, S. L. J. Am. Chem. Soc. 1994, 116, 11797-11810.

(s10) Smith, M. B.; Guo, L. C.; Okeyo, S.; Stenzel, J.; Yanella, J.; LaChapelle, E. Org. Lett. 2002, 4, $2321-2323$. 


\section{General procedure for the iodination of aromatic amines.}

To a solution of aromatic amine $4(2.4 \mathrm{mmol})$ in anhydrous diethyl ether $(32 \mathrm{~mL})$ was added dropwise, at $-78^{\circ} \mathrm{C}$ under argon, a solution of $n$-butyllithium $(2.5 \mathrm{M}$ in hexanes, $0.96 \mathrm{~mL}, 2.4$ mmol). The resulting mixture was stirred for $1 \mathrm{~h}$ at $-78^{\circ} \mathrm{C}$ and then added to the dry polymer $3 \mathbf{a}$ $(2.07 \mathrm{~g}, 2.4 \mathrm{mmol})$. The resulting heterogeneous mixture was stirred for $18 \mathrm{~h}$ at room temperature before adding a solution of $\mathrm{ICl}(3.6 \mathrm{mmol})$ in diethyl ether $(10 \mathrm{~mL})$ at $-78^{\circ} \mathrm{C}$. The mixture was allowed to warm to room temperature and stirred for $24 \mathrm{~h}$ in the dark before filtration of the polymer. The resin was at first washed with diethyl ether $(6 \times 40 \mathrm{~mL})$, then with THF $(6 \times 40$ $\mathrm{mL})$ and absolute ethanol $(4 \times 40 \mathrm{~mL})$, and dried in vacuo $(0.5 \mathrm{mbar})$ at $60^{\circ} \mathrm{C}$ for $5 \mathrm{~h}$ before reuse. Polymer $3 \mathbf{c}$ was obtained as a brown resin and was found to contain $1.36 \mathrm{mmol}$ of $\mathrm{Sn}-$ Cl/g. Anal. Found: C, 68.99; H, 7.71; Sn, 16.12; Cl, 5.52; I, $<0.20 .{ }^{119} \mathrm{Sn}$ MAS NMR (186.5 $\mathrm{MHz}) \delta_{\mathrm{Sn}} 170$ ppm; IR (KBr) v 3082, 3058, 3025, 2920, 2851, 1942, 1870, 1800, 1719, 1601, $1586,1493,1452,1071,1028,759,698 \mathrm{~cm}^{-1}$. The ethereal filtrate was then successively washed with a saturated solution of $\mathrm{NaHCO}_{3}(50 \mathrm{~mL})$ and brine $(50 \mathrm{~mL})$, dried over $\mathrm{MgSO}_{4}$, and concentrated in vacuo. The crude product was purified by chromatography on silica gel.

\section{Characterization of 4-iodoarylamines 8a-h}

All compounds 8a-h were obtained following the general procedure for the iodination of aromatic amines.

4-iodoaniline 8a. ${ }^{\text {s11 }}$ Compound $8 \mathbf{a}$ was obtained from aniline $4 \mathbf{a}(2.43 \mathrm{mmol})$ as a brown solid after chromatography (9:1, petroleum ether/AcOEt, $\left.1 \% \mathrm{Et}_{3} \mathrm{~N}, \mathrm{Rf}=0.15\right)$ : $324 \mathrm{mg}, 61 \%$; mp 64$65^{\circ} \mathrm{C} ;{ }^{1} \mathrm{H}$ NMR $\left(300 \mathrm{MHz}, \mathrm{CDCl}_{3}\right) \delta_{\mathrm{H}} 3.68(\mathrm{br} \mathrm{s}, 2 \mathrm{H}), 6.45-6.50(\mathrm{~m}, 2 \mathrm{H}), 7.38-7.43(\mathrm{~m}, 2 \mathrm{H}) ;{ }^{13} \mathrm{C}$ NMR $\left(75.5 \mathrm{MHz}, \mathrm{CDCl}_{3}\right) \delta_{\mathrm{C}} 79.4,117.3,137.9,146.0 ; \mathrm{MS}-\mathrm{EI} \mathrm{m} / z$ (relative intensity) 219 (100), 127 (5), 109.5 (6), 92 (58), 65 (57), 63 (14); IR (KBr) v 3405, 3300, 3200, 3058, 3028, 1629, $1583,1484,1275,816 \mathrm{~cm}^{-1}$.

4-iodo-2-nitroaniline $8 \mathrm{~b}^{\text {s12 }}$ Compound $8 \mathrm{~b}$ was obtained from 2-nitroaniline $4 \mathbf{b}(2.4 \mathrm{mmol})$ as an orange solid after chromatography $\left(8: 2\right.$, petroleum ether/AcOEt, $\left.1 \% \mathrm{Et}_{3} \mathrm{~N}, \mathrm{Rf}=0.31\right): 399 \mathrm{mg}$, 63\%; mp 121-122 ${ }^{\circ}$; ${ }^{1} \mathrm{H}$ NMR (300 MHz, $\left.\mathrm{CDCl}_{3}\right) \delta_{\mathrm{H}} 6.09$ (br s, 2H), $6.61(\mathrm{~d}, J=8.7 \mathrm{~Hz}, 1 \mathrm{H})$, $7.56(\mathrm{dd}, J=8.7 \mathrm{~Hz}, J=2.1 \mathrm{~Hz}, 1 \mathrm{H}), 8.43(\mathrm{~d}, J=2.1 \mathrm{~Hz}, 1 \mathrm{H}) ;{ }^{13} \mathrm{C} \mathrm{NMR}\left(75.5 \mathrm{MHz}, \mathrm{CDCl}_{3}\right) \delta_{\mathrm{C}}$

(s11) Edgar, K. J.; Falling, S. N. J. Org. Chem. 1990, 55, 5287-5291.

(s12) Price, D. W., Jr.; Dirk, S. M.; Maya, F.; Tour, J. M. Tetrahedron 2003, 59, 2497-2518. 
75.9, 120.6, 133.1, 134.3, 143.7, 144.0; MS-EI $m / z$ (relative intensity) 264 (100), 234 (4), 218 (27), 127 (5), 91 (55), 90 (15), 64 (21), 63 (24), 52 (20); IR (KBr) v 3491, 3379, 1614, 1610, $1554,1491,1460,1335,1249,1232,811 \mathrm{~cm}^{-1}$.

2-fluoro-4-iodoaniline 8d. ${ }^{\text {s13 }}$ Compound 8d was obtained from 2-fluoroaniline 4d (2.4 mmol) as a pale red solid after chromatography $\left(9: 1\right.$, petroleum ether/AcOEt, $\left.1 \% \mathrm{Et}_{3} \mathrm{~N}, \mathrm{Rf}=0.29\right): 379 \mathrm{mg}$, 67\%; mp 52-53 ${ }^{\circ} \mathrm{C} ;{ }^{1} \mathrm{H}$ NMR $\left(300 \mathrm{MHz}, \mathrm{CDCl}_{3}\right) \delta_{\mathrm{H}} 3.74$ (br s, $\left.2 \mathrm{H}\right), 6.53$ (dd, $J=8.4 \mathrm{~Hz}, J=9.0$ $\mathrm{Hz}, 1 \mathrm{H}), 7.21(\mathrm{ddd}, J=1.8 \mathrm{~Hz}, J=8.4 \mathrm{~Hz}, J=0.9 \mathrm{~Hz}, 1 \mathrm{H}), 7.28(\mathrm{dd}, J=1.8 \mathrm{~Hz}, J=10.5 \mathrm{~Hz}$, $1 \mathrm{H}) ;{ }^{13} \mathrm{C} \mathrm{NMR}\left(75.5 \mathrm{MHz}, \mathrm{CDCl}_{3}\right) \delta_{\mathrm{C}} 77.3,118.4,124.1(\mathrm{~d}, J=21.1 \mathrm{~Hz}), 133.3,134.5(\mathrm{~d}, J=$ $12.8 \mathrm{~Hz}$ ), 149.8-153.1 (d, $J=243.9 \mathrm{~Hz}$ ); MS-EI m/z (relative intensity) 237 (100), 127 (4), 118.5 (6), 110 (48), 90 (24), 83 (41), 63 (16), 57 (14); IR (KBr) v3418, 3330, 3213, 1622, 1575, 1494, $1409,1291,1204,871,817 \mathrm{~cm}^{-1}$.

1-amino-4-iodonaphtalene $\mathbf{8 h}$. Compound $\mathbf{8 h}$ was obtained from 1-aminonaphtalene $\mathbf{4 h}(2.4$ $\mathrm{mmol})$ as a red solid after chromatography $\left(85: 15\right.$, petroleum ether/AcOEt, $\left.1 \% \mathrm{Et}_{3} \mathrm{~N}, \mathrm{Rf}=0.37\right)$ : $286 \mathrm{mg}, 44 \%$; mp 82-83 ${ }^{\circ} \mathrm{C}$; ${ }^{1} \mathrm{H}$ NMR $\left(300 \mathrm{MHz}, \mathrm{CDCl}_{3}\right) \delta_{\mathrm{H}} 4.20$ (br s, $2 \mathrm{H}$ ), 6.56 (d, J=7.8 Hz, 1H), 7.46-7.58 (m, 2H), 7.75 (d, $J=9.0 \mathrm{~Hz}, 1 \mathrm{H}), 7.83$ (d, $J=7.8 \mathrm{~Hz}, 1 \mathrm{H}), 8.05$ (d, $J=9.0 \mathrm{~Hz}$, $1 \mathrm{H}) ;{ }^{13} \mathrm{C} \mathrm{NMR}\left(75.5 \mathrm{MHz}, \mathrm{CDCl}_{3}\right) \delta_{\mathrm{C}} 85.7,111.2,121.3,124.6,125.6,127.7,132.8,134.5$, 137.5, 143.2; MS-EI $m / z$ (relative intensity) 269 (100), 142 (31), 140 (10), 134.5 (4), 115 (33); IR $(\mathrm{KBr}) v 3448,3364,3047,1595,1566,1512,1385,1301,1138,823,758 \mathrm{~cm}^{-1}$.

(s13) Glendenning, M. E., Goodby, J. W., Hird, M., Toyne, K. J. J. Chem. Soc., Perkin Trans. 2 1999, $481-491$. 